\title{
Assessing the impact of pneumococcal conjugate vaccines on invasive pneumococcal disease using polymerase chain reaction-based surveillance: an experience from South Africa
}

\author{
Stefano Tempia ${ }^{1,2,3^{*}}$ D, Nicole Wolter ${ }^{3,4}$, Cheryl Cohen ${ }^{3,5}$, Sibongile Walaza ${ }^{3,5}$, Claire von Mollendorf ${ }^{3,5}$,
}

Adam L. Cohen ${ }^{1,2}$, Jocelyn Moyes ${ }^{3,5}$, Linda de Gouveia ${ }^{3}$, Susan Nzenze ${ }^{5,6}$, Florette Treurnicht ${ }^{3}$, Marietjie Venter ${ }^{7}$, Michelle J. Groome ${ }^{6,8}$, Shabir A. Madhi ${ }^{3,6,8}$ and Anne von Gottberg ${ }^{3,4^{*}}$

\begin{abstract}
Background: The use of molecular diagnostic techniques for the evaluation of the impact of pneumococcal conjugate vaccines (PCVs) has not been documented. We aimed to evaluate the impact of PCVs on invasive pneumococcal disease (IPD) using polymerase chain reaction (PCR)-based techniques and compare with results obtained from culture-based methods.
\end{abstract}

Methods: We implemented two independent surveillance programs for IPD among individuals hospitalized at one large surveillance site in Soweto, South Africa during 2009-2012: (i) PCR-based (targeting the lytA gene) syndromic pneumonia surveillance; and (ii) culture-based laboratory surveillance. Positive samples were serotyped. The molecular serotyping assay included targets for 42 serotypes including all serotypes/serogroups included in the 7-valent (PCV-7) and 13-valent (PCV-13) PCV. The Quellung reaction was used for serotyping of culture-positive cases. We calculated the change in rates of IPD (IytA- or culture-positive) among HIV-uninfected children aged $<2$ years from the year of PCV-7 introduction (2009) to the post-vaccine years (2011 or 2012).

Results: During the study period there were 607 lytA-positive and 1,197 culture-positive cases that were serotyped. Samples with lytA cycle threshold (Ct)-values $\geq 35$ (30.2\%; 123/407) were significantly less likely to have a serotype/ serogroup detected for serotypes included in the molecular serotyping assay than those with Ct-values $<35$ (78.0\%; 156/200) ( $p<0.001$ ). From 2009 to 2012 rates of PCV-7 serotypes/serogroups decreased $-63.8 \%$ (95 \% Cl: $-79.3 \%$ to $-39.1 \%)$ among lytA-positive cases and $-91.7 \%$ (95 \% Cl: $-98.8 \%$ to $-73.6 \%)$ among culture-positive cases. Rates of lytA-positive non-vaccine serotypes/serogroups also significantly decreased $(-71.7 \%$; $95 \% \mathrm{Cl}:-81.1 \%$ to $-58.5 \%)$ over the same period. Such decline was not observed among the culture-positive non-vaccine serotypes $(1.2 \% ; 95 \% \mathrm{Cl}:-96.7 \%$ to $58.4 \%)$.

(Continued on next page)

*Correspondence: stefanot@nicd.ac.za; annev@nicd.ac.za

1 Influenza Division, Centers for Disease Control and Prevention, Atlanta, Georgia, USA

${ }^{3}$ Centre for Respiratory Diseases and Meningitis, National Institute for Communicable Diseases of the National Health Laboratory Service, Johannesburg, South Africa

Full list of author information is available at the end of the article

C 2015 Tempia et al. Open Access This article is distributed under the terms of the Creative Commons Attribution 4.0 international License (http://creativecommons org/licenses/by/40/ which permits unrestricted use distribution, and reproduction in any medium, provided you give appropriate credit to the original author(s) and the source, provide a link to the Creative Commons license, and indicate if changes were made. The Creative Commons Public Domain Dedication waiver (http://creativecommons.org/publicdomain/zero/1.0/) applies to the data made available in this article, unless otherwise stated. 
(Continued from previous page)

Conclusions: Significant downward trends in IPD PCV-7 serotype-associated rates were observed among patients tested by PCR or culture methods; however trends of non-vaccine serotypes/serogroups differed between the two groups. Misclassifications of serotypes/serogroups, affecting the use of non-vaccine serotypes as a control group, may have occurred due to the low performance of the serotyping assay among lytA-positive cases with high Ctvalues. Until PCR methods improve further, culture methods should continue to be used to monitor the effects of PCV vaccination programs on IPD incidence.

Keywords: Pneumococcus, Conjugate vaccine, lytA, Molecular serotyping, South Africa

\section{Background}

Every year pneumococcal disease results in $\approx 600,000$ deaths among children $<5$ years of age globally, with the majority of deaths occurring in Africa [1]. While over 90 Streptococcus pneumoniae serotypes have been identified [2], approximately 20 are responsible for the majority of invasive pneumococcal disease (IPD) [3]. The direct and indirect effects of the pneumococcal conjugate vaccines (PCVs), which target the most common serotypes associated with IPD, have been documented in several highincome countries [4-7].

In 2009, South Africa introduced the 7-valent PCV (PCV-7) into its routine infant immunization program using a schedule of vaccination at 6 and 14 weeks and a booster dose at 9 months [4]. PCV-7 was replaced by the 13-valent PCV (PCV-13) in April 2011 [4]. The benefit of the introduction of PCV-7 and subsequently PCV-13 have been documented in South Africa using data from a nationwide, laboratory-based IPD surveillance program [4]. IPD cases were detected through the identification of S. pneumoniae from cultured specimens that were subsequently serotyped using the Quellung reaction [5].

The determination of pneumococcal serotypes is key to assess the effects of PCVs, including decreases in PCV serotypes and potential non-PCV serotype replacement following the use of the vaccine over time. With PCVs being progressively introduced into the routine infant immunization programs of several low- and middleincome countries [6], serotype-specific pneumococcal surveillance is key to assess the impact of the vaccine in diverse settings.

Culture remains the gold standard for the identification of the organism while the Quellung reaction remains the gold standard for serotype determination from available isolates. Nonetheless, culture, while highly specific, has low sensitivity, requires long incubation periods and is not commonly available in many low-income countries [7]. In addition, antibiotic therapy prior to specimen collection or suboptimal culturing conditions may reduce the yield of cultures $[8,9]$.

Polymerase chain reaction (PCR)-based methods targeting pneumococcal specific genes, such as $l y t A$, have resulted in improved and timely diagnosis of pneumococcal diseases [10-12]. Such methods can be easily implemented where molecular diagnostic capacity exists and could become an alternative diagnostic tool in settings where culture capacity is lacking or suboptimal. Nonetheless, the use of molecular diagnostic techniques for the evaluation of the impact of PCVs against IPD has not been documented.

We aimed to evaluate the impact of PCVs on IPD using PCR-based methods at one large surveillance site in South Africa from 2009 through 2012, and compare these results with those obtained from culture-based methods.

\section{Methods}

Description of the surveillance programs The Severe Acute Respiratory IIIness (SARI) program (PCR-based syndromic surveillance)

We conducted active, prospective, syndromic, hospitalbased surveillance at Chris Hani-Baragwanath Academic Hospital (CHBAH) from February 2009 through December 2012. This hospital is the only public hospital serving a well-defined community (Soweto, Gauteng Province) of about 1.4 million people in 2012 [13] from which rates of hospitalizations can be estimated [14, 15]. We aimed to test all enrolled individuals with $l y t A$ real-time PCR on whole blood. For the SARI program a case of bacteremic pneumococcal pneumonia (BPP) was defined as the identification of S. pneumoniae in blood specimens using a single-target (lytA) quantitative real-time PCR assay adapted from Carvalho et al. [16]. lytA-positive specimens (cycle threshold $(\mathrm{Ct}$ )-value $<40$ ) were serotyped by realtime PCR using an adaption of the method described by Azzari et al. [17]. The molecular serotyping assay included targets for 42 serotypes including all serotypes/serogroups included in PCV-7 and PCV-13 PCV (see Supplementary Material for Additional file 1). DNA extraction was performed using the Roche MagNA Pure LC 1.0 instrument during May 2009-January 2010, the Roche MagNA Pure LC 2.0 instrument during February 2010-July 2012 and the Roche MagNA Pure 96 instrument during AugustDecember 2012. 
The Group for Enteric, Respiratory and Meningeal Disease Surveillance (GERMS) program (culture-based laboratory surveillance)

Data on active, laboratory-based IPD surveillance conducted under the GERMS program at CHBAH were included in this study. For the GERMS program, IPD cases were defined as hospitalized persons from whom $S$. pneumoniae was cultured from specimens that are normally sterile (e.g., cerebrospinal fluid (CSF), blood or joint fluid). Cultures were taken as clinically indicated by attending clinical staff. Strains were serotyped by the Quellung reaction targeting 93 serotypes [5].

The study and laboratory procedures of the SARI and GERMS programs have been previously described $[4,14$, $15,18]$ and are summarized in Additional file 1. While the SARI and GERMS surveillance programs were implemented independently, co-enrolment of patients was possible. This would have been in instances when a patient tested positive for S. pneumoniae on culture locally, but also meet the SARI case definition. In addition, while the GERMS program only enrolled patients with $S$. pneumoniae-positive culture results, results of any blood culture (including negative results) taken routinely on-site were collected under the SARI program. These culture were taken as clinically indicated by attending clinical staff. Fig. 1 provides the enrolment of cases under the SARI and GERMS programs, including coenrolment.

Written informed consent was obtained from all cases who were 18 years of age and older. Proxy informed consent was obtained from parents or legal guardians of minors.

\section{Statistical analysis}

We implemented a 3-stage analysis whereby stage-1 and -2 analyses were conducted to inform the interpretation of results of the main analysis in stage 3. The analytical approach for each analysis is described in Additional file 1. The analysis was implemented using Stata 13.1 (StataCorp ${ }^{\oplus}$, Texas, USA).

\section{Stage-1 analysis: proportion of serotypable samples by lytA} Ct-value among lytA-positive patients with SARI

In the stage- 1 analysis we evaluated the proportion of serotypable samples by lyt $\mathrm{Ct}$-value among the lyt $A$ positive SARI samples obtained from patients of any age (Fig. 1). We conducted this analysis because we suspected that lytA-positive samples with high $\mathrm{Ct}$-values would be associated with a low performance of the molecular serotyping assay as previously reported [19].

\section{Stage-2 analysis: factors associated with increasing Ct- values among lytA-positive patients with SARI}

In the stage-2 analysis we evaluated factors associated with increasing $\mathrm{Ct}$-values among lytA-positive SARI patients of any age (Fig. 1). We conducted this analysis because in the stage-1 analysis we observed a low performance of the serotyping assay for $l y t A$-positive samples with high $\mathrm{Ct}$-values. Variable performance of the serotyping assay could impact the interpretation of the trend analysis of BPP cases by vaccine serotypes

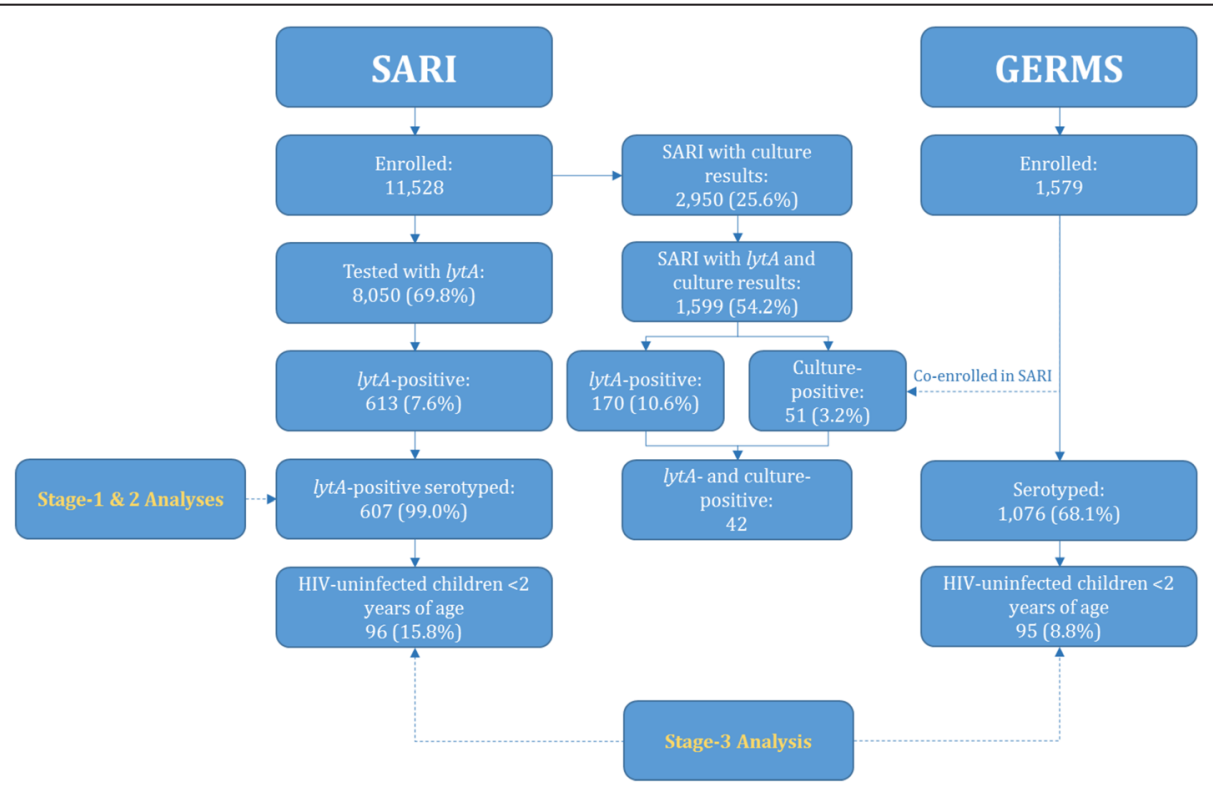

Fig. 1 Enrolment of cases with severe acute respiratory illness (SARI program) and cases of culture-positive invasive pneumococcal disease (GERMS program) hospitalized at Chris Hani-Baragwanath Academic Hospital, Soweto, South Africa, 2009-2012 
(stage-3 analysis). In particular, a variation in the proportion of lytA-positive samples with higher or lower Ctvalues over time could result in varying proportions of serotypable samples affecting the observed trends of BPP by vaccine serotype.

\section{Stage-3 analysis: time-trends of BPP (lytA-positive) and IPD (culture-positive) among HIV-uninfected children $<2$ years of age}

The aim of the study was to assess the feasibility of evaluating the impact of PCVs using PCR-based methods, and therefore, for the main analysis we focused on HIV-uninfected children $<2$ years of age (Fig. 1). This group was chosen because it is directly vaccinated and the effectiveness of PCV has been well documented in several countries [20-23], including South Africa [4].

To assess the trends of BPP over time we calculated the annual rate of $l y t A$-positive SARI hospitalizations overall and by PCV-7, additional PCV-13 and nonvaccine serotypes/serogroups during 2009-2012. We assessed the impact of the introduction of PCV-7 and PCV-13 by calculating the reduction in rates of BPP (expressed as percentage reduction with associated $95 \%$ confidence intervals) between the post-vaccine years (2011 or 2012) and the year of introduction of PCV (2009). A similar trend analysis was implemented using the culture-positive cases. Rates were expressed per 100,000 person-years.

\section{Ethical approval}

The SARI protocol was approved by the University of the Witwatersrand Human Research Ethics Committee (M081042) and the University of KwaZulu-Natal Biomedical Research Ethics Committee (BF157/08). The GERMS protocol was approved by the research ethics committee of the University of the Witwatersrand (M081117).

\section{Results}

\section{Description of SARI cases}

From February 2009 through December 2012, 8,050/ 11,528 (69.8\%) of SARI cases enrolled at CHBAH were tested for whole blood $l y t A$, of which 613 (7.6\%) were lytA-positive (Fig. 1). The lytA-detection rate varied by age: $4.9 \%$ (130/2639), $5.5 \%$ (24/438), $8.3 \%$ (44/530), $9.5 \%(269 / 2841), 10.2 \%(135 / 1321)$ and $2.7 \%(7 / 258)$ among individuals $<2,2-4,5-24,25-44,45-64$ and $\geq 65$ years of age, respectively $(\mathrm{p}<0.001)$. The lyt $A$-detection rate varied also by year: $8.0 \%(129 / 1616)$ in 2009 , $7.5 \%(173 / 2293)$ in 2010, $6.3 \%(152 / 2421)$ in 2011 and $9.2 \%(159 / 1720)$ in $2012(p=0.005)$. In 2012, the lytAdetection rate was higher among samples from which DNA was extracted using the Roche MagNA Pure 96 instrument (14.8\%; 73/492) than using the Roche MagNA Pure LC 2.0 instrument (7.0\%; 86/1228) $(p<$ 0.001).

Of the 613 lytA-positive cases, 607 (99.0\%) were tested with the serotyping assay and were included for further analyses. The HIV serostatus was known for 558/ 607 (91.9 \%) individuals of which 395 (70.8 \%) were HIV positive. The HIV prevalence varied by age: $11.9 \%$ (13/ 109), $20.0 \%$ (4/20), $82.1 \%$ (32/39), $94.1 \%$ (240/255), $81.1 \%(103 / 127)$ and $33.3 \%(2 / 6)$ among individuals $<2$, $2-4,5-24,25-44,45-64$ and $\geq 65$ years of age, respectively $(p<0.001)$.

A culture result was available for 2,950/11,528 (25.6\%) SARI cases, of which 69 (2.3\%) tested positive for $S$. pneumoniae. Among the 1599 SARI cases with both lytA and culture results available, $179(11.2 \%)$ tested positive in at least one of the assays. Of these, 170 (95.0\%) and $51(28.5 \%)$ specimens tested positive for $l y t A$ and culture, respectively; 128 (71.5\%) cases tested positive for lytA alone, 9 (5.0 \%) for culture alone and 42 (23.5\%) for both $l y t A$ and culture. The detection rate was $10.6 \%$ $(170 / 1599)$ and $3.2 \%(51 / 1599)$ for lyt $A$ and culture, respectively $(p<0.001)$.

Among the 607/613 (99.0 \%) lytA-positive samples that were tested with the serotyping assay, 166 (27.3\%) had available culture results and $42(25.3 \%)$ tested positive for S. pneumoniae; $16 / 29$ (55.2 \%), 11/33 (33.3\%) and $15 / 104$ (14.4 \%) among samples with lytA Ct-value of $\leq 30,31-34$ and $\geq 35$, respectively ( $<<0.001$ ). Among the 42 cases that tested positive in both assays, a serotype could be identified in 36 (85.7\%) cases; 32 (76.2 \%) cases using the Quellung reaction and 26 (61.9\%) cases using the molecular serotyping assay. A serotype could be identified by both assays in 22/36 (61.1 \%) cases. Among these, the same serotype/serogroup was identified by both assays in 21 (95.5\%) cases. A serotype could be identified by the Quellung reaction, but not by the molecular serotyping assay in 10/36 (27.8\%) cases. Of these, 8 (80.0\%) were serotypes/serogroups included in the molecular serotyping assay, of which 7 (87.5\%) had a $l y t A$ Ct-value $\geq 35$ and $1(12.5 \%)$ had a lytA Ctvalue of 34 . All of them were PCV-7, PCV-13 or $6 \mathrm{~A}$ serotypes. A serotype/serogroup could be identified by the molecular serotyping assay, but not by the Quellung reaction in $4 / 36(11.1 \%)$ cases. The characteristics of the 36 cases for which a serotype/serogroup was identified are provided in Table 1.

\section{Stage-1 analysis: proportion of serotypable samples by lytA Ct-value among lytA-positive patients with SARI}

Of the 607 lytA-positive SARI samples that were tested with the serotyping assay, a serotype/serogroup included in the assay was detected in 279 (46.0 \%) samples. Among these, the most frequently detected serotypes/ 
Table 1 Characteristics of S. pneumoniae-positive cases $(N=36)$ hospitalized at Chris Hani Baragwanath Academic Hospital for which a serotype/serogroup could be identified by the Quellung reaction and/or the molecular serotyping assay, Soweto, South Africa, 2009-2012

\begin{tabular}{|c|c|c|c|c|}
\hline \multirow[t]{2}{*}{ Age group(in years) } & \multirow[t]{2}{*}{ HIV serostatus } & \multirow[t]{2}{*}{ lytACt-value } & \multicolumn{2}{|l|}{ Serotype/serogroup } \\
\hline & & & Quellung reaction & Molecular serotyping assay \\
\hline \multicolumn{5}{|l|}{ lytA Ct-value $\leq 30$} \\
\hline $25-44$ & Pos & 25 & $19 \mathrm{~F}$ & 19B/19 F \\
\hline $45-64$ & Pos & 26 & 1 & 1 \\
\hline$<2$ & Pos & 27 & $10 A^{c}$ & $\mathrm{Neg} 42$ \\
\hline $25-44$ & Pos & 27 & $19 \mathrm{~F}$ & $19 B / F$ \\
\hline $25-44$ & Unknown & 27 & Not available ${ }^{b}$ & $18 \mathrm{~A} / \mathrm{B} / \mathrm{C}$ \\
\hline $25-44$ & Pos & 27 & $19 A$ & $19 \mathrm{~A}$ \\
\hline $25-44$ & Pos & 28 & $19 A$ & $19 \mathrm{~A}$ \\
\hline $25-44$ & Pos & 28 & $19 \mathrm{~F}$ & 19B/F \\
\hline $25-44$ & Pos & 29 & $19 A$ & $19 \mathrm{~A}$ \\
\hline $25-44$ & Pos & 29 & $19 A$ & $19 A$ \\
\hline $25-44$ & Pos & 30 & $19 A$ & $19 \mathrm{~A}$ \\
\hline \multicolumn{5}{|l|}{ lytA Ct-value 31-34 } \\
\hline $25-44$ & Pos & 31 & 3 & 3 \\
\hline $45-64$ & Unknown & 31 & $19 A$ & $19 A$ \\
\hline $25-44$ & Pos & 31 & 1 & 1 \\
\hline $25-44$ & Pos & 32 & $12 \mathrm{~F}$ & $12 \mathrm{~A} / \mathrm{B} / \mathrm{F}$ \\
\hline $25-44$ & Neg & 32 & $19 \mathrm{~A}$ & $19 \mathrm{~A}$ \\
\hline $45-64$ & Pos & 33 & 4 & 4 \\
\hline $25-44$ & Pos & 33 & Not available & $19 \mathrm{~A}$ \\
\hline $25-44$ & Neg & 34 & 1 & 1 \\
\hline $25-44$ & Pos & 34 & Not available & 1 \\
\hline $25-44$ & Pos & 34 & $1^{d}$ & $\mathrm{Neg} 42$ \\
\hline $45-64$ & Pos & 34 & $19 \mathrm{~A}$ & $19 \mathrm{~A}$ \\
\hline \multicolumn{5}{|l|}{ lyt A Ct-value $\geq 35$} \\
\hline $5-24$ & Pos & 35 & $9 \mathrm{~V}$ & 9A/L/N/N \\
\hline$<2$ & Pos & 35 & $23 \mathrm{~F}$ & $23 \mathrm{~F}$ \\
\hline $5-24$ & Pos & 35 & $19 A^{d}$ & $\mathrm{Neg} 42$ \\
\hline $25-44$ & Pos & 35 & $1^{d}$ & $\mathrm{Neg} 42$ \\
\hline$<2$ & Neg & 36 & $6 \mathrm{~A}$ & $6 \mathrm{~A} / \mathrm{B}$ \\
\hline$<2$ & Unknown & 36 & $6 B$ & $6 \mathrm{~A} / \mathrm{B}$ \\
\hline $5-24$ & Pos & 36 & $18 C^{d}$ & Neg42 \\
\hline $25-44$ & Pos & 37 & $19 A^{d}$ & $\mathrm{Neg} 42$ \\
\hline $2-4$ & Neg & 37 & $14^{\mathrm{d}}$ & Neg42 \\
\hline $25-44$ & Pos & 37 & Not available & 1 \\
\hline $25-44$ & Pos & 38 & $1^{d}$ & $\mathrm{Neg} 42$ \\
\hline
\end{tabular}


Table 1 Characteristics of S. pneumoniae-positive cases $(N=36)$ hospitalized at Chris Hani Baragwanath Academic Hospital for which a serotype/serogroup could be identified by the Quellung reaction and/or the molecular serotyping assay, Soweto, South Africa, 2009-2012 (Continued)

\begin{tabular}{|c|c|c|c|c|}
\hline $25-44$ & Pos & 38 & $23 A^{c}$ & Neg42 \\
\hline $2-4$ & Pos & 39 & $6 A^{d}$ & Neg42 \\
\hline$<2$ & Neg & 39 & $19 A^{e}$ & $18 \mathrm{~A} / \mathrm{B} / \mathrm{C}^{\mathrm{e}}$ \\
\hline
\end{tabular}

Abbreviations: HIV: human immunodeficiency virus; Ct-value: cycle threshold value; Neg42: samples that tested negative for the 42 serotypes detected by the serotyping assay

a Discrepant or missing serotype/serogroup results are in bolt font

${ }^{b}$ Isolate not available for serotyping using the Quellung reaction

c Serotype not included in the molecular serotyping assay

d Serotype included in the molecular serotyping assay

serogroups were 19A (61; $21.8 \%), 1(52 ; 18.6 \%)$ and 6A/B (33; $11.8 \%)$. The lytA Ct-value ranged between 25 and 39 (median 36). We observed a decline of the proportion of serotypable samples among samples with an individual $l y t A$ Ct-value $\geq 34$ (Fig. 2 and Table 2). However, compared to samples with Ct-values $\leq 30$ this decline was statistically significant among samples with individual Ct-values $\geq 35$ (Table 2). The proportion of serotypable samples declined from $76.1 \%(54 / 71)$ among samples with Ct-value $\leq 30$ to $15.7 \%(8 / 51)$ among samples with Ct-value of $39(\mathrm{p}<0.001)$. Overall, the proportion of samples with Ct-value $<34$ or $<35$ was $26.0 \%$ (158/607) and $32.9 \%$ (200/607), respectively.

\section{Stage-2 analysis: factors associated with increasing Ct-values among lytA-positive patients with SARI}

Among the 607 lytA-positive SARI samples that were tested with the serotyping assay, 71 (11.7 \%) had Ctvalues $\leq 30,129(21.2 \%)$ had Ct-values $31-34$ and

Table 2 Proportion of serotypable $e^{a}$ lytA-positive samples ( $n=607$ ) by lytA cycle threshold value (Ct-value) among patients hospitalized with severe acute respiratory illness at Chris Hani-Baragwanath Academic Hospital, Soweto, South Africa, 2009-2012

\begin{tabular}{llll}
\hline IytA Ct-value & \multicolumn{3}{l}{ Serotypable lytA-positive samples } \\
\cline { 2 - 4 } & $\mathrm{n} / \mathrm{N}(\%)$ & OR $(95 \% \mathrm{Cl})$ & $\mathrm{p}$ \\
\hline$\leq 30$ & $54 / 71(76.1)$ & Reference & - \\
31 & $20 / 23(86.9)$ & $2.1(0.6-7.9)$ & 0.275 \\
32 & $22 / 28(78.6)$ & $1.2(0.4-3.3)$ & 0.790 \\
33 & $32 / 36(88.9)$ & $2.5(0.8-8.1)$ & 0.123 \\
34 & $28 / 42(66.7)$ & $0.6(0.3-1.5)$ & 0.281 \\
35 & $31 / 57(54.4)$ & $0.4(0.2-0.8)$ & 0.011 \\
36 & $29 / 72(40.3)$ & $0.2(0.1-0.4)$ & $<0.001$ \\
37 & $28 / 93(30.1)$ & $0.1(0.07-0.3)$ & $<0.001$ \\
38 & $27 / 134(20.1)$ & $0.08(0.04-0.15)$ & $<0.001$ \\
39 & $8 / 51(15.7)$ & $0.06(0.02-0.14)$ & $<0.001$ \\
\hline
\end{tabular}

Abbreviations: OR: odds ratio; $\mathrm{Cl}$ : confidence interval a Serotypable samples were samples tested with the serotyping assay from which a serotype/serogroup included in the assay was detected
407 (67.1 \%) had Ct-values $\geq 35$. On multivariable analysis (Table 3 ), factors negatively associated with increasing lytA Ct-values were: (i) extraction instrument Roche MagNA Pure LC 2.0 (adjusted odds ratio [aOR]: $0.4 ; 95 \%$ confidence intervals [CI]: 0.2-0.6) or Roche MagNA Pure 96 (aOR: 0.3; 95 \% CI: 0.1-0.7) compared to Roche MagNA Pure LC 1.0; (ii) HIV infection (aOR: 0.4; $95 \%$ CI: 0.2-0.7); (iii) duration of hospitalization for 3-7 days (aOR: 0.4; 95 \% CI: $0.2-$ 0.8 ) or $\geq 8$ days (aOR: 0.3 ; $95 \%$ CI: 0.1-0.5) compared to $0-2$ days; and (iv) in-hospital death (aOR: 0.3; 95 \% CI: 0.2-0.6). Additional PCV-13 serotypes/serogroups were significantly less associated with increasing lytA Ct-values (aOR: 0.3; 95 \% CI: 0.2-0.5), while non-vaccine serotypes/serogroups were significantly more associated with increasing lyt $\mathrm{A} \mathrm{Ct}$-values (aOR: 2.7 ; 95 \% CI: 1.6-4.6) compared with PCV-7 serotypes/serogroups (Table 3 ).

\section{Stage-3 analysis: time-trends of BPP (lytA-positive) and IPD (culture-positive) among HIV-uninfected children $<2$ years of age}

The proportion of PCV serotypes/serogroups among lytA-positive (SARI) and culture-positive (GERMS) cases is provided in Additional file 1 (Table S1 and Figure S1). Overall from 2009 to 2012, among HIVuninfected children $<2$ years of age a reduction in rates of $-64.0 \%$ (95\% CI: $-72.9 \%$ to $-52.6 \%$ ) was observed among lytA-positive cases compared to $-66.8 \%$ (95 \% CI: $-81.2 \%$ to $-43.8 \%$ ) among culture-positive cases (Table 4). Over the same period, rates of PCV-7 serotypes/serogroups decreased $-63.8 \%$ (95\% CI: $-79.3 \%$ to $-39.1 \%$ ) among lytApositive cases and $-91.7 \%$ (95 \% CI: $-98.4 \%$ to $-73.6 \%)$ among culture-positive cases. Rates of $l y t A$ positive non-vaccine serotypes/serogroups also significantly decreased $(-71.7 \%$; $95 \%$ CI: $-81.1 \%$ to $-58.5 \%)$ over the same period. Such decline was not observed among the culture-positive non-vaccine serotypes $(1.2 \%$; $95 \% \mathrm{CI}:-96.7 \%$ to $58.4 \%)$. Among lytA-positive cases the time-trends of non-vaccine 


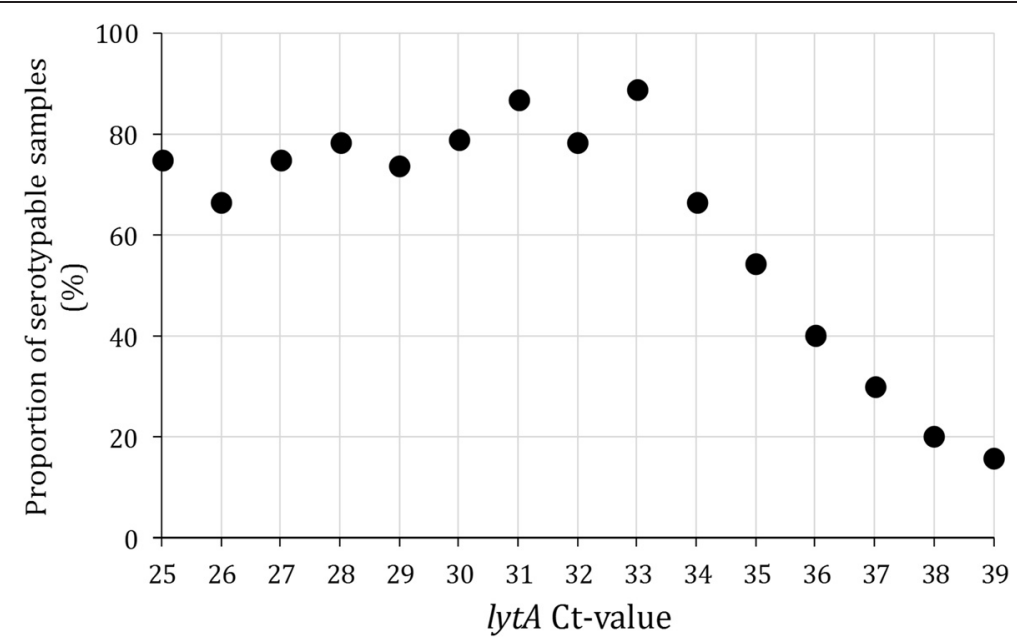

Fig. 2 Proportion of serotypable lytA-positive samples $(n=607)$ by lytA cycle threshold value (Ct-value) among patients hospitalized with severe acute respiratory illness at Chris Hani-Baragwanath Academic Hospital, Soweto, South Africa, 2009-2012. Serotypable samples were samples tested with the serotyping assay from which a serotype/serogroup included in the assay was detected

serotypes/serogroups mimicked closely those of PCV7 serotypes/serogroups and the rates of non-vaccine serotypes/serogroups were consistently higher than those of PCV-7 and PCV-13 serotypes/serogroups even during the year of vaccine introduction (2009) (Fig. 3a). This was not observed for culture-positive cases (Fig. 3b).

An increase in rates of $l y t A$-positive cases was observed from 2011 to 2012 for all PCV categories (Table 4 and Fig. 3a), while this was not observed among culturepositive cases for which declines in PCV-7 and PCV-13 were observed (Table 4 and Fig. 3b).

From 2009 to 2011, the time-trends and the proportional decrease in rates of PCV-7 serotypes/serogroups was similar among lytA-positive (-80.9\%; $95 \%$ CI: $-90.9 \%$ to $-62.9 \%)$ and culture-positive (-83.2 \%; $95 \%$ CI: $-94.2 \%$ to $-59.5 \%$ ) cases (Table 4 and Fig. $3 a$ and b). The sharpest decline of PCV-7 serotypes/serogroups was observed from 2009 to 2010 for both lytA- (Fig. 3a) and culture-positive cases (Fig. 3b). Among lytA-positive cases a sharper decline was observed between 2009 and 2010 for non-vaccine serotypes/serogroups (-76.8\%; 95 \% CI: -81.3 \% to $-69.2 \%)$ compared to PCV-7 serotypes/serogroups $(-53.2 \% ; 95 \%$ CI: $\quad-64.7 \%$ to $-41.6 \%)$.

\section{Discussion}

We expected that the introduction of PCV into our national immunization program would lead to declines in pneumococcal disease, especially vaccinetype disease among the vaccinated population. This has been shown from surveillance data using traditional culture-based methods [4, 20-23], and the expectation was that this would also be seen in surveillance using newer molecular techniques. Overall, using both PCR- and culture-based methods we reported a significant decline of BPP or IPD rates during the early years of PCV-7 introduction among HIV-uninfected children $<2$ years of age in Soweto. Nonetheless, the PCR-based results would have been difficult to interpret in the absence of culture-based data because molecular methods showed a decline in vaccine-type as well as non-vaccine-type disease and laboratory testing results were sensitive to the bacterial load and equipment used.

As expected and previously reported [4], rates of lytAand culture-positive PCV-7 serotypes/serogroups significantly declined over the study period (stage-3 analysis), probably owing to the progressive effect of the introduction of PCV-7 into the routine infant immunization program. Nevertheless, rates of lytA-positive non-vaccine serotypes, which should not be impacted by the use of PCV-7, unexpectedly also significantly decreased and were consistently higher than those of PCV-7 and PCV13 over the study period. Consistent with previous studies [20-23], including data from South Africa [4], this was not observed for culture-positive cases.

In the stage- 1 analysis, we observed a significant reduction of the proportion of serotypable samples (i.e., positive for one of the serotypes/serogroups detected by the serotyping assay) among lytA-positive cases with $\mathrm{Ct}$ values $\geq 35$ as previously reported [19]. The low performance of the serotyping assay among lytA-positive cases with high $\mathrm{Ct}$-values could result in the misclassification of the PCV serotypes/serogroups as non-vaccine types (non-vaccine serotypes/serogroups including samples negative for the 42 serotypes detected by the serotyping assay: Neg42) as observed among cases with available 
Table 3 Factors associated with increasing lytA cycle threshold value (Ct-value) among lytA-positive patients hospitalized with severe acute respiratory illness at Chris Hani-Baragwanath Academic Hospital, Soweto, South Africa, 2009-2012

\begin{tabular}{|c|c|c|c|c|c|c|c|c|}
\hline \multirow[t]{3}{*}{ Variable } & \multirow{2}{*}{\multicolumn{4}{|c|}{ lytA Ct-value }} & \multicolumn{4}{|c|}{ Proportional-Odds Model } \\
\hline & & & & & \multicolumn{2}{|c|}{ Univariate analysis } & \multicolumn{2}{|c|}{ Multivariable analysis } \\
\hline & $\overline{\text { Totaln (\%) }}$ & $\leq 30 n(\%)$ & $31-34 n(\%)$ & $\geq 35 n(\%)$ & $\mathrm{OR}^{\mathrm{b}}(95 \% \mathrm{Cl})$ & $\overline{p \text {-value }}$ & $\mathrm{aOR}^{\mathrm{b}}(95 \% \mathrm{Cl})$ & $p$-value \\
\hline Age (in years) & $N=603$ & $N=71$ & $N=128$ & $N=404$ & & & & \\
\hline$<2$ & $125(20.7)$ & $6(8.5)$ & $11(8.6)$ & $108(26.7)$ & Reference & - & & \\
\hline $2-4$ & $24(4.0)$ & $0(0.0)$ & $6(4.7)$ & $18(4.5)$ & $0.5(0.2-1.5)$ & 0.227 & & \\
\hline $5-24$ & $44(7.3)$ & $2(2.8)$ & $13(10.2)$ & $29(7.2)$ & $0.3(0.2-0.7)$ & 0.008 & & \\
\hline $25-44$ & $268(44.4)$ & $48(67.6)$ & $59(46.1)$ & $161(39.8)$ & $0.2(0.1-0.4)$ & $<0.001$ & & \\
\hline $45-64$ & $135(22.4)$ & $14(19.7)$ & $37(28.9)$ & $84(20.8)$ & $0.3(0.1-0.5)$ & $<0.001$ & & \\
\hline$\geq 65$ & $7(1.2)$ & $1(1.4)$ & $2(1.5)$ & $4(1.0)$ & $0.2(0.1-1.1)$ & $<0.051$ & & \\
\hline Sex & $N=603$ & $N=71$ & $N=128$ & $N=404$ & & & & \\
\hline Male & $257(42.6)$ & $27(38.0)$ & $64(50.0)$ & $166(41.1)$ & Reference & - & & \\
\hline Female & $346(57.4)$ & $44(62.0)$ & $64(50.0)$ & $238(58.9)$ & 1.1 & 0.453 & & \\
\hline Year & $N=607$ & $N=71$ & $N=129$ & $N=407$ & & & & \\
\hline 2009 & $129(21.3)$ & $8(11.3)$ & $18(13.9)$ & $103(25.3)$ & Reference & - & & \\
\hline 2010 & $173(28.5)$ & $34(47.9)$ & $46(35.7)$ & $93(22.8)$ & $0.3(0.2-0.5)$ & $<0.001$ & & \\
\hline 2011 & $150(24.7)$ & $10(14.1)$ & $39(30.2)$ & $101(24.8)$ & $0.6(0.3-0.9)$ & 0.033 & & \\
\hline 2012 & $155(25.5$ & $19(26.8)$ & $26(20.2)$ & $110(27.0)$ & $0.6(0.3-1.1)$ & 0.064 & & \\
\hline Extraction Instrument & $N=607$ & $N=71$ & $N=129$ & $N=407$ & & & & \\
\hline Roche MagNA Pure LC 1.0 & $136(22.4)$ & $9(12.7)$ & $19(14.7)$ & $108(26.5)$ & Reference & - & Reference & - \\
\hline Roche MagNA Pure LC 2.0 & $400(65.9)$ & $54(76.1)$ & $96(74.4)$ & $250(61.4)$ & $0.4(0.3-0.7)$ & $<0.001$ & $0.4(0.2-0.6)$ & $<0.001$ \\
\hline Roche MagNA Pure 96 & $71(11.7)$ & $8(11.3)$ & $14(10.8)$ & $49(12.0)$ & $0.6(0.3-1.1)$ & 0.092 & $0.3(0.1-0.7)$ & 0.004 \\
\hline Antibiotics $24 \mathrm{H}$ before admission & $N=601$ & $N=71$ & $N=128$ & $N=402$ & & & & \\
\hline No & $567(94.3)$ & $68(95.8)$ & $122(95.3)$ & $377(93.8)$ & Reference & - & & \\
\hline Yes & $34(5.7)$ & $3(4.2)$ & $6(4.7)$ & $25(6.2)$ & $1.4(0.6-3.0)$ & 0.393 & & \\
\hline Antibiotics during admission & $N=586$ & $N=69$ & $N=126$ & $N=391$ & & & & \\
\hline No & $19(3.2)$ & $3(4.3)$ & $2(1.6)$ & $14(3.6)$ & Reference & - & & \\
\hline Yes & $567(96.8)$ & $66(95.6)$ & $124(98.4)$ & $377(96.4)$ & $0.8(0.3-2.2)$ & 0.647 & & \\
\hline Underlying medical conditions ${ }^{a}$ & $N=603$ & $N=71$ & $N=128$ & $N=404$ & & & & \\
\hline No & $565(93.7)$ & $66(93.0)$ & $120(93.7)$ & $379(93.8)$ & Reference & - & & \\
\hline Yes & $38(6.3)$ & $5(7.0)$ & $8(6.3)$ & $25(6.2)$ & $0.9(0.5-1.8)$ & 0.839 & & \\
\hline HIV infection & $N=558$ & $N=66$ & $N=119$ & $N=373$ & & & & \\
\hline No & $163(29.2)$ & $5(7.6)$ & $22(18.5)$ & $136(36.5)$ & Reference & - & Reference & - \\
\hline Yes & $395(70.8)$ & $61(92.4)$ & $97(81.5)$ & $237(63.5)$ & $0.3(0.2-0.5)$ & $<0.001$ & $0.4(0.2-0.7)$ & 0.001 \\
\hline PCV serotypes/serogroups & $N=607$ & $N=71$ & $N=129$ & $N=407$ & & & & \\
\hline PCV-7 & $111(18.3)$ & $13(18.3)$ & $28(21.7)$ & $70(17.2)$ & Reference & - & Reference & - \\
\hline PCV-13 & $138(22.7)$ & $35(49.3)$ & $62(48.1)$ & $41(10.1)$ & $0.3(0.2-0.5)$ & $<0.001$ & $0.3(0.2-0.5)$ & $<0.001$ \\
\hline $\mathrm{NVT}$ & $358(59.0)$ & $23(32.4)$ & $39(30.2)$ & $296(72.7)$ & $2.7(1.7-4.4)$ & $<0.001$ & $2.7(1.6-4.6)$ & $<0.001$ \\
\hline Duration of symptoms (in days) & $N=602$ & $N=71$ & $N=127$ & $N=404$ & & & & \\
\hline $0-2$ & $204(33.9)$ & $14(19.7)$ & $38(29.9)$ & $152(37.6)$ & Reference & - & & \\
\hline$\geq 3$ & $398(66.1)$ & $57(80.3)$ & $89(70.1)$ & $252(62.4)$ & $0.6(0.4-0.8)$ & 0.003 & & \\
\hline Duration of hospitalization (in days) & $N=602$ & $N=71$ & $N=129$ & $N=402$ & & & & \\
\hline $0-2$ & $92(15.3)$ & $1(1.4)$ & 15 (11.6) & 76 918.9) & Reference & - & Reference & - \\
\hline $3-7$ & $267(44.3)$ & 26 (36.6) & $61(47.3)$ & $180(44.8)$ & $0.4(0.2-0.8)$ & 0.004 & $0.5(0.2-1.1)$ & 0.071 \\
\hline
\end{tabular}


Table 3 Factors associated with increasing lytA cycle threshold value (Ct-value) among lytA-positive patients hospitalized with severe acute respiratory illness at Chris Hani-Baragwanath Academic Hospital, Soweto, South Africa, 2009-2012 (Continued)

\begin{tabular}{|c|c|c|c|c|c|c|c|c|}
\hline$\geq 8$ & $243(40.4)$ & $44(62.0)$ & $53(41.1)$ & $146(36.3)$ & $0.3(0.1-0.5)$ & $<0.001$ & $0.3(0.1-0.6)$ & 0.002 \\
\hline In-hospital outcome & $N=603$ & $N=71$ & $N=129$ & $N=403$ & & & & \\
\hline Survived & $562(93.2)$ & $58(81.7)$ & $119(92.2)$ & $385(95.5)$ & Reference & - & Reference & - \\
\hline Died & $41(6.8)$ & $13(18.31)$ & $10(7.8)$ & $18(4.5)$ & $0.3(0.2-0.6)$ & $<0.001$ & $0.3(0.2-0.7)$ & 0.003 \\
\hline
\end{tabular}

Abbreviations: OR: odds ratio; aOR: adjusted odds ratio; Cl: confidence interval; HIV: human immunideficency virus; PCV-7: 7-valent pneumococcal conjugate vaccine serotypes (included serotypes/serogroups 4, 6A/B, 9A/V/L/N, 14, 18A/B/C, 19B/F, 23 F); PCV-13: additional 13-valent pneumococcal conjugate vaccine serotypes (included serotypes/serogroups 1,3,5,7A/F, 19A); NVT: serotypes/serogroups not included in PCV-7 or PCV-13, including samples that tested negative for the 42 serotypes detected by the serotyping assay

a Underlying medical conditions included: asthma, chronic lung disease, chronic heart disease, liver disease, renal disease, diabetes mellitus, immunocompromizing conditions excluding HIV infection or neurological disease

${ }^{b}$ The odds ratio of the proportional-odds model measures the effect of a predictor on the odds of being above a specified level, compared with the odds of being at or below the specified level

serotype results from both molecular- and culture-based methods. This could potentially explain the high rates and the downward trends observed in the non-vaccine serotype group (stage-3 analysis). The significant positive association of non-vaccine compared to PCV-7 serotypes/serogroups with increasing $\mathrm{Ct}$-values (stage- 2 analysis) increases the plausibility of this hypothesis. In addition, the fact that the time-trends of non-vaccine serotypes/serogroups among any lytA-positive cases (Ctvalue $<40)$ mimicked closely those of PCV-7 serotypes/ serogroups (stage- 3 analysis) further suggests that, while lytA-positive samples with Ct-values $\geq 35$ could not be accurately serotyped and hence were classified as Neg42, they were probably true cases that included misclassified PCV serotypes/serogroups.

Of note is that the proportion of vaccine and nonvaccine serotypes/serogroups was similar among culture-positive and lytA-positive cases with Ct-value
$<35$ (Additional file1). This further suggests that more reliable molecular serotype results can be obtained from samples with lyt $A$ Ct-values $<35$ as observed in the stage1 analysis. In addition, it appears that non-vaccine serotypes potentially included in the PCV-7 $(9 \mathrm{~A} / \mathrm{L} / \mathrm{N}, 18 \mathrm{~A} / \mathrm{B}$ and 19B) and PCV-13 (7A) categories (as a result of potential misclassification of non-vaccine serotypes as vaccine serotypes within serogroups) did not significantly alter the proportion of vaccine and non-vaccine categories compared to serotype-specific culture results. The nonvaccine serotypes potentially misclassified as vaccine types in this study accounted for $<1 \%$ of the overall burden of IPD in previous studies conducted in South Africa [4].

Nonetheless, in our study only $\approx 33 \%$ of all lytA-positive samples had a Ct-value $<35$, hindering our ability to implement a trend analysis using more conservative Ctvalue cut-offs, especially when focusing on specific age and HIV-serostatus groups. While the overall number of

Table 4 Rates of bacteremic pneumococcal pneumonia (SARI program - lytA-positive) and invasive pneumococcal pneumonia (GERMS program - culture-positive) among HIV-uninfected children <2 years of age hospitalized at Chris Hani-Baragwanath Academic Hospital, Soweto, South Africa, 2009-2012.

\begin{tabular}{|c|c|c|c|c|c|c|c|}
\hline \multirow{3}{*}{$\begin{array}{l}\text { PCV } \\
\text { serotypes }\end{array}$} & \multicolumn{3}{|c|}{ Hospitalization rates per 100,000 person-years } & \multicolumn{4}{|c|}{ Relative difference in hospitalization rates } \\
\hline & \multirow{2}{*}{$\begin{array}{l}2009 \\
\text { Rate (95 \% Cl) }\end{array}$} & \multirow{2}{*}{$\begin{array}{l}2011 \\
\text { Rate (95 \% Cl) }\end{array}$} & \multirow{2}{*}{$\begin{array}{l}2012 \\
\text { Rate (95\% Cl) }\end{array}$} & \multicolumn{2}{|l|}{2009 to 2011} & \multicolumn{2}{|l|}{2009 to 2012} \\
\hline & & & & $\%(95 \% \mathrm{Cl})$ & $p$ & $\%(95 \% \mathrm{Cl})$ & $p$ \\
\hline \multicolumn{8}{|c|}{ Any lytA-positive (SARI program) } \\
\hline PCV-7 & $125.1(93.7-163.6)$ & $23.9(11.9-42.8)$ & $45.2(28.0-69.1)$ & $-80.9(-90.9$ to -62.9$)$ & $<0.001$ & $-63.8(-79.3$ to -39.1$)$ & $<0.001$ \\
\hline PCV-13 & $37.8(21.6-61.3)$ & $15.2(6.1-31.3)$ & $34.4(19.7-55.9)$ & $-59.7(-85.9$ to +3.4$)$ & 0.067 & $+8.8(-94.8$ to +57.3$)$ & 0.796 \\
\hline NVT & $273.7(226.2-328.3)$ & $47.8(30.0-72.4)$ & $77.5(54.3-107.3)$ & $-82.5(-89.4$ to -72.3$)$ & $<0.001$ & $-71.7(-81.1$ to -58.5$)$ & $<0.001$ \\
\hline All & $436.6(375.9-504.2)$ & $86.9(62.1-118.3)$ & $157.1(123.2-197.6)$ & $-80.1(-86.2$ to -71.8$)$ & $<0.001$ & $-64.0(-72.9$ to -52.6$)$ & $<0.001$ \\
\hline \multicolumn{8}{|c|}{ Culture-positive (GERMS program) } \\
\hline PCV-7 & $77.9(53.6-109.3)$ & $13.0(4.8-28.4)$ & $6.5(1.3-18.9)$ & $-83.2(-94.2$ to -59.5$)$ & $<0.001$ & $-91.7(-98.4$ to -73.6$)$ & $<0.001$ \\
\hline PCV-13 & $23.6(11.3-43.4)$ & $17.4(7.5-34.2)$ & $8.6(2.3-22.1)$ & $-26.3(-74.7$ to +107.3$)$ & 0.529 & $-63.5(-91.6$ to +26.5$)$ & 0.084 \\
\hline $\mathrm{NVT}$ & $28.3(14.6-49.5)$ & $28.2(15.0-48.3)$ & $28.0(14.9-47.8)$ & $-0.2(-58.0$ to +139.2$)$ & 0.993 & $+1.2(-96.7$ to +58.4$)$ & 0.974 \\
\hline All & $129.8(97.8-168.9)$ & $58.6(38.7-85.3)$ & $43.1(26.3-66.5)$ & $-54.8(-72.6$ to -27.1$)$ & $<0.001$ & $-66.8(-81.2$ to -43.8$)$ & $<0.001$ \\
\hline
\end{tabular}

Abbreviations: PCV-7: 7-valent pneumococcal conjugate vaccine serotypes (included serotypes/serogroups 4, 6A/B, 9A/V/L/N, 14, 18A/B/C, 19B/F, 23 F for lyApositive samples and 4, 6A/B, $9 \mathrm{~V}, 14,18 \mathrm{C}, 19 \mathrm{~F}, 23 \mathrm{~F}$ for culture-positive samples); PCV-13: additional 13-valent pneumococcal conjugate vaccine serotypes (included serotypes/serogroups 1, 3, 5, 7A/F, 19A for lyA-positive samples and 1, 3, 5, $7 \mathrm{~F}$, 19A for culture-positive samples); NVT: serotypes/serogroups not included in PCV-7 or PCV-13, including samples that tested negative for the 42 serotypes detected by the serotyping assay for lytA-positive samples 

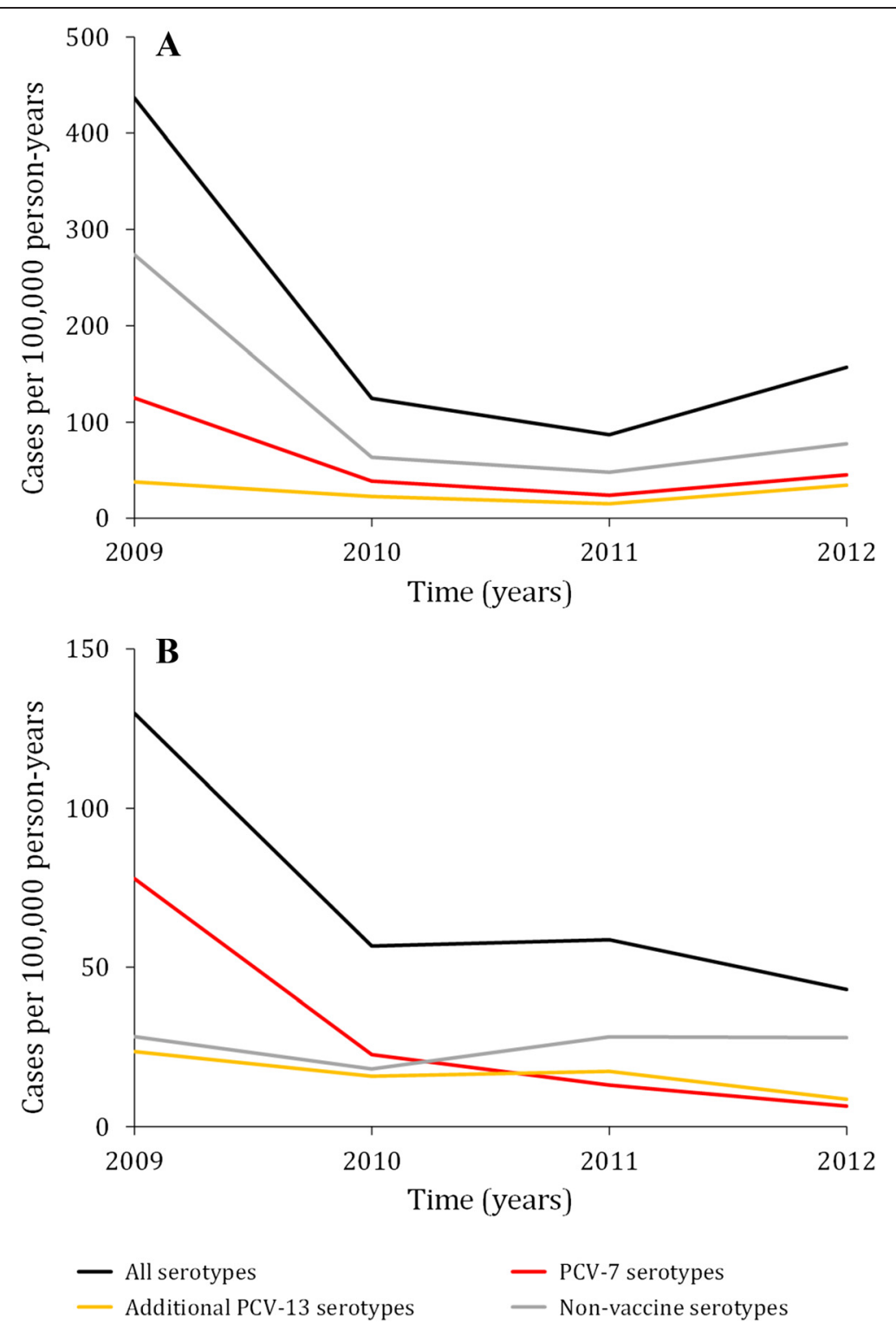

Fig. 3 Rates of invasive S. pneumoniae-associated hospitalizations among HIV-uninfected children <2 years of age at Chris-Hani Baragwanath Academic Hospital, Soweto, South Africa, 2009-2012. a: lytA-positive cases (SARI program) (7-valent pneumococcal conjugate vaccine (PCV-7) serotypes/serogroups included: 4, 6A/B, 9AN/L/N, 14, 18A/B/C, 19B/F, 23 F; additional 13-valent pneumococcal conjugate vaccine (PCV-13) serotypes/serogroups included: 1, 3, 5, 7A/F, 19A). b: culture-positive cases (GERMS program) (7-valent pneumococcal conjugate vaccine (PCV-7) serotypes included: 4, 6A/B, 9 V, 14, 18C, 19 F, 23 F; additional 13-valent pneumococcal conjugate vaccine (PCV-13) serotypes included: 1, 3, 5, 7 F, 19A). Non-vaccine serotypes included serotypes/serogroups not included in PCV-7 or PCV-13, including samples that tested negative for the 42 serotypes detected by the serotyping assay for lytA-positive samples

lytA-positive cases obtained in our study was well above the number of cases needed to significantly estimate a decline in PCV serotypes using population based methods [24], this was not the case when restricting the analysis to lytA-positive cases with $\mathrm{Ct}$-value $<35$.

A significantly higher decline of non-vaccine compared to PCV-7 serotypes/serogroups was observed from 2009 to 2010 potentially owing to the combined effect of the reduction of PCV-7 serotypes/serogroups misclassified in the non-vaccine category as well as the use of the
Roche MagNA Pure LC 2.0 extraction instrument from February 2010. In the stage- 2 analysis the use of the Roche MagNA Pure LC 2.0 compared to the Roche MagNA Pure LC 1.0 instrument for DNA extraction was significantly less associated with increasing lytA Ctvalues. This suggests that the use of a better extraction instrument would increase the proportion of lytA-positive samples with low Ct-values and consequently increase their likelihood to be correctly serotyped using the serotyping assay (stage-1 analysis). This would result 
in improved classifications of PCV-7 and PCV-13 serotype/serogroups (increasing rates in these categories) and consequently reduced misclassification of the same serotype/serogroups (decreasing rates in the non-vaccine category) in 2010 compared to 2009. The replacement of the MagNA Pure LC 2.0 instrument with the Roche MagNA Pure 96 instrument in August 2012 could also have introduced bias in the trend analysis. In 2012, the detection rate of $l y t A$-positive cases doubled following the introduction of the new instrument potentially resulting in the rate increase observed from 2011 to 2012, whereas this was not observed among culturepositive cases. This highlights the importance of standardization of procedures over time for time-trend analysis purposes. Nonetheless, while the standardization of methods across the study period is key to avoid the introduction of biases, this may conflict with the use of rapidly evolving technology and the need to upgrade laboratory equipment over time.

In the stage- 2 analysis, besides the use of different extraction instruments and the non-vaccine serotypes/ serogroups, factors negatively associated with increasing Ct-values were HIV infection and in-hospital death. The lyt $A \mathrm{Ct}$-value provides a semi-quantitative measure of the pneumococcal load, with lower Ctvalues indicative of higher load and vice-versa. The association of high pneumococcal load among lytApositive cases with HIV infection and in-hospital deaths has been previously reported [18].

Among lytA-positive samples with available culture results the proportion of culture-positive samples decreased with increasing lyt $A \mathrm{Ct}$-values, and was only $\approx$ $55 \%$ even among lytA-positive samples with Ct-values $\leq 30$. This highlights the usefulness of the use of PCRbased methods for improved diagnosis of pneumococcal disease as previously reported [10-12].

Our study has limitations that warrant discussion. First, we did not have lytA data for years prior to the introduction of PCV-7 and our data were limited to one large surveillance site in the country. Nonetheless, data from nationwide culture-based surveillance reported downward trends of IPD PCV-7-associated rates [4] similar to those reported in this analysis. Second, we did not systematically test all enrolled patients using PCRand culture-based methods hindering our ability to directly compare results from the same group of patients. Nonetheless, the proportion of vaccine and non-vaccine type disease was similar between syndromes (i.e., meningitis, bacteremic pneumonia and bacteremia without focus) among South African children $<5$ years of age during the pre-vaccine era [25] and there was no statistically significant difference in the proportion of vaccineand non-vaccine-type disease between blood- and CSFpositive specimens among HIV-uninfected children
$<2$ years of age in this study. Last, the molecular serotyping assay targets only 42 serotypes/serogroups leaving uncertainty about the lytA-positive samples that tested negative for the 42 targets.

\section{Conclusions}

In conclusion, in our setting the overall downward trends in IPD PCV-7 serotypes-associated rates were similar among patients tested with PCR- or culturebased methods; however trends of non-vaccine serotypes/serogroups differed between the two groups. While PCR-based methods could be used to assess trends of PCV-7 serotypes/serogroups the misclassifications observed in this study affected the use of nonvaccine types as a control group. Such misclassifications could also potentially hinder the ability to assess serotype replacement following the use of PCVs over time. These findings suggest that current molecular methods alone may not be sufficient to monitor the impact of PCV unless standardized procedures and equipment are used throughout the study period and large populations are systematically surveyed to allow time-trend analysis using more restrictive Ct-value cut-offs. If the results of this study are confirmed in other settings, the development of improved molecular serotyping assays would enhance serotype-specific pneumococcal surveillance using PCR-based methods. Improvements of the molecular serotyping assays would entail increased sensitivity and inclusion of targets for all serotypes/serogroups.

\section{Ethics}

The SARI protocol was approved by the University of the Witwatersrand Human Research Ethics Committee (M081042) and the University of KwaZulu-Natal Biomedical Research Ethics Committee (BF157/08). The GERMS protocol was approved by the research ethics committee of the University of Witwatersrand and by local hospitals or provincial ethics committees as required.

\section{Additional file}

Additional file 1: Assessing the Impact of Pneumococcal Conjugate Vaccines on Invasive Pneumococcal Disease Using Polymerase Chain Reaction-Based Surveillance: An Experience from South Africa (Supplementary Material). (DOCX $65 \mathrm{~kb}$ )

\section{Abbreviations}

BPP: Bacteremic Pneumococcal Pneumonia; CHBAH: Chris Hani-Baragwanath Academic Hospital; CSF: Cerebrospinal Fluid; Ct-value: Cycle Threshold Value; GERMS: Group for Enteric, Respiratory and Meningeal Disease Surveillance; IPD: Invasive Pneumococcal Diseases; PCR: Polymerase Chain Reaction; PCV: Pneumococcal Conjugate Vaccine; PCV-13: 13-Valent Pneumococcal Conjugate Vaccine; PCV-7: 7-Valent Pneumococcal Conjugate Vaccine; S. pneumoniae: Streptococcus pneumoniae; SARI: Severe Acute Respiratory Illness. 


\section{Competing interests}

No authors have any competing interests.

\section{Authors' contribution}

ST contributed to the study concept and design, acquisition and interpretation of data, critical revision of the manuscript for important intellectual content, drafted the manuscript and implemented the statistical analysis. NW, CC and AvG contributed to the study concept and design, acquisition and interpretation of data and critical revision of the manuscript for important intellectual content. SW, CvM, JM, LdG and MJG contributed to the acquisition and interpretation of data and critical revision of the manuscript for important intellectual content. ALC contributed to the study concept and design and critical revision of the manuscript for important intellectual content. SN, FT, MV and SAM contributed to the critical revision of the manuscript for important intellectual content. All authors read and approved the final version of the manuscript.

\section{Acknowledgments}

We thank all laboratory and clinical staff throughout South Africa for contributing to national surveillance of invasive pneumococcal disease as well as all members involved in the severe acute respiratory illness surveillance program for the collection of specimens and management of data.

\section{Disclaimer}

The findings and conclusions in this report are those of the authors and do not necessarily represent the official position of the US Centers for Disease Control and Prevention or the National Institute for Communicable Diseases.

\section{Financial disclosure}

This work was supported by Pfizer South Africa (investigator-initiated research agreement number: WS1167521) and the US Centers for Disease Control and Prevention (co-operative agreement number: 5U51IP000155).

\begin{abstract}
Author details
${ }^{1}$ Influenza Division, Centers for Disease Control and Prevention, Atlanta, Georgia, USA. ${ }^{2}$ Influenza Program, Centers for Disease Control and Prevention, Pretoria, South Africa. ${ }^{3}$ Centre for Respiratory Diseases and Meningitis, National Institute for Communicable Diseases of the National Health Laboratory Service, Johannesburg, South Africa. ${ }^{4}$ School of Pathology, Faculty of Health Sciences, University of the Witwatersrand, Johannesburg, South Africa. ${ }^{5}$ School of Public Health, Faculty of Health Sciences, University of the Witwatersrand, Johannesburg, South Africa. ${ }^{6}$ Medical Research Council, Respiratory and Meningeal Pathogens Research Unit, University of the Witwatersrand, Johannesburg, South Africa. ${ }^{7}$ Division of Global Health Protection, Centers for Disease Control and Prevention, Pretoria, South Africa. ${ }^{8}$ Department of Science and Technology/National Research Foundation: Vaccine Preventable Diseases, University of the Witwatersrand, Johannesburg, South Africa.
\end{abstract}

Received: 17 June 2015 Accepted: 8 October 2015

Published online: 26 October 2015

\section{References}

1. Naghavi M, Wang H, Lozano R, Davis A, Liang X, Zhou M, et al. Global, regional, and national age-sex specific all-cause and cause specific mortality for 240 causes of death, 1990-2013: a systematic analysis for the global burden of disease study 2013. Lancet. 2015;385(9963):117-71.

2. Calix JJ, Porambo RJ, Brady AM, Larson TR, Yother J, Abeygunwardana C, et al. Biochemical, genetic, and serological characterization of two capsule subtypes of Streptococcus pneumoniae serotype 20 strains: discovery of a new pneumococcal serotype. J Biol Chem. 2012;287(33):27885-94.

3. Johnson HL, Deloria-Knoll M, Levine OS, Stoszek SK, Freimanis Hance L, Reithinger R, et al. Systematic evaluation of serotypes causing invasive pneumococcal disease among children under five: the pneumococcal global serotype project. PLoS Med. 2010;7:10.

4. von Gottberg A, de Gouveia L, Tempia S, Quan V, Meiring S, von Mollendorf $C$, et al. Effects of vaccination on invasive pneumococcal disease in South Africa. N Engl J Med. 2014;371(20):1889-99.
5. Austrian R. The Quellung reaction, a neglected microbiologic technique. Mt Sinai J Med. 1976;43(6):699-709.

6. Centers for Disease Control and Prevention (CDC). Progress in introduction of pneumococcal conjugate vaccine - worldwide, 2000-2012. MMWR Morb Mort Wkly Rep. 2013;62(16):308-11.

7. Frean J, Perovic O, Fensham V, McCarthy K, von Gottberg A, de Gouveia L, et al. External quality assessment of national public health laboratories in Africa, 2002-2009. Bull World Health Organ. 2012;90(3):191-199A.

8. Nigrovic LE, Malley R, Macias CG, Kanegaye JT, Moro-Sutherland DM, Schremmer RD, et al. Effect of antibiotic pretreatment on cerebrospinal fluid profiles of children with bacterial meningitis. Pediatrics. 2008;122(4):726-30.

9. Resti M, Micheli A, Moriondo M, Becciolini L, Cortimiglia M, Canessa C, et al. Comparison of the effect of antibiotic treatment on the possibility of diagnosing invasive pneumococcal disease by culture or molecular methods: a prospective, observational study of children and adolescents with proven pneumococcal infection. Clin Ther. 2009:31(6):1266-73.

10. Azzari C, Moriondo M, Indolfi G, Massai C, Becciolini L, de Martino M, et al. Molecular detection methods and serotyping performed directly on clinical samples improve diagnostic sensitivity and reveal increased incidence of invasive disease by Streptococcus pneumoniae in Italian children. J Med Microbiol. 2008:57(Pt 10):1205-12.

11. Benson R, Tondella ML, Bhatnagar J, Carvalho Mda G, Sampson JS, Talkington DF, et al. Development andevaluation of a novel multiplex PCR technology for molecular differential detection of bacterial respiratory disease pathogens. J Clin Microbiol. 2008;46(6):2074-7.

12. Carvalhanas TR, Ribeiro AF, Kemp B, Salgado MM, Shutt KA, Carvalhanas TR, et al. Incorporation of real-time PCR into routine public health surveillance of culture negative bacterial meningitis in Sao Paulo, Brazil. PLoS One. 2011;6(6):e20675.

13. Statistics South Africa. Population statistics. http://www.statssa.gov.za/ ?page_id=1854\&PPN=P0302. Accessed 15 January 2015.

14. Cohen C, Moyes J, Tempia S, Groom M, Walaza S, Pretorius M, et al. Severe influenza-associated lower respiratory tract infection in a high HIVPrevalence setting - South Africa, 2009-2011. Emerg Infec Dis. 2013;19(11):1766-74.

15. Moyes J, Cohen C, Pretorius M, Groome M, von Gottberg A, Wolter N, et al. Epidemiology of respiratory syncytial virus-associated acute lower respiratory tract infection hospitalizations among HIV-infected and HIV-uninfected South African children, 2010-2011. J Infect Dis. 2013;208(S3):S217-26.

16. Carvalho M, Tondella ML, McCaustland K, Groome M, von Gottberg A, Wolter $\mathrm{N}$, et al. Evaluation of improvement of real-time PCR assays targeting IytA, ply, and psaA genes for detection of pneumococcal DNA. J Clin Microbiol. 2007:45(8):2460-6.

17. Azzari C, Moriondo M, Indolfi G, Cortimiglia M, Canessa C, Becciolini L, et al. Real-time PCR is more sensitive than multiplex PCR for diagnosis and serotyping in children with culture negative pneumococcal invasive disease. PLoS One. 2010;5(2):e9282.

18. Wolter N, Cohen C, Tempia S, Madhi SA, Venter M, Moyes J, et al. HIV and influenza virus infection are associated with increased blood pneumococcal load: a prospective hospital-based observational study in South Africa, 2009-2011. J Infcet Dis. 2014;209(1):56-65.

19. Magomani V, Wolter N, Tempia S, du Plessis M, de Gouveia L, von Gottberg $A$, et al. Challenges of using molecular serotyping for surveillance of pneumococcal disease. J Clin Microbiol. 2014;52(9):3271-6.

20. Pilishvili T, Lexau C, Farley MM, Hadler J, Harrison LH, Bennett NM, et al. Sustained reductions in invasive pneumococcal disease in the era of conjugate vaccine. J Infect Dis. 2010;201(1):32-41.

21. Miller E, Andrews NJ, Waight PA, Slack MP, George RC. Herd immunity and serotype replacement 4 years after seven-valent pneumococcal conjugate vaccination in England and Wales: an observational cohort study. Lancet Infect Dis. 2011;11(10):760-8.

22. Centers for Disease Control and Prevention (CDC). Direct and indirect effects of routine vaccination of children with 7-valent pneumococcal conjugate vaccine on incidence of invasive pneumococcal disease United States 1998-2003. MMWR Morb Mortal Wkly Rep. 2005;54(36):893-7.

23. Cohen AL, Harrison LH, Farley MM, Reingold AL, Hadler J, Schaffner W, et al. Prevention of invasive pneumococcal disease among HIV-infected 
adults in the era of childhood pneumococcal immunization. AIDS 2010;24(14):2253-62.

24. Hampton LM, Zell ER, Schrag S, Cohen AL. Sentinel versus population-based surveillance of pneumococcal conjugate vaccine effectiveness. Bull World Health Organ. 2012;90(8):568-77.

25. von Gottberg A, Cohen C, de Gouveia L, Meiring S, Quan V, Whitelaw A, et al. Epidemiology of invasive pneumococcal disease in the pre-conjugate vaccine era: South Africa, 2003-2008. Vaccine. 2013:31(38):4200-8.

Submit your next manuscript to BioMed Central and take full advantage of:

- Convenient online submission

- Thorough peer review

- No space constraints or color figure charges

- Immediate publication on acceptance

- Inclusion in PubMed, CAS, Scopus and Google Scholar

- Research which is freely available for redistribution 